\title{
Effect of Aerobic Physical Exercise and Training Status on the Perceived Egocentric Distances
}

\author{
Mohamed Jarraya ${ }^{* *}$, Hamdi Chtourou ${ }^{*}, \quad$ Nizar Souissi*, Karim Chamari* \\ "Research Laboratory "Sports performance optimization" National Center of \\ Medicine and Science in Sports (CNMSS), Tunis, Tunisia. \\ ${ }^{*}$ Research Unit, High Institute of Sport and Physical Education, Sfax, Tunisia. \\ E-mail: jarrayam@yahoo.fr, h_chtourou@yahoo.fr, n_souissi@yahoo.fr, \\ karimchamari@gmx.net
}

\begin{abstract}
This study was to investigate the effect of moderateintensity-exercise and training status on the perception of the egocentric distance (PED). To this end, 18 footballers $(23 \pm 0.5 \mathrm{yrs} ; 72 \pm 3.8 \mathrm{~kg}$; $1.73 \pm 2.5 \mathrm{~m})$ and 18 sedentary subjects $(23 \pm 0.2 \mathrm{yrs}$; $69 \pm 4.2 \mathrm{~kg} ; 1.71 \pm 2.8 \mathrm{~m}$ ) participated in this study. The subjects have carried out a distance perception task while pedalling on an ergocycle for $10 \mathrm{~min}$ at an intensity of $60 \%$ of the maximal-aerobic-power. The estimations were recorded during rest-time, phase 1 (1 to $2 \mathrm{~min}$ ), phase 2 (5 to $6 \mathrm{~min}$ ), phase 3 (9 to $10 \mathrm{~min}$ ), and after the effort. The results showed that the precision of PED was greater in trained than untrained subjects $(p<0.05)$ and during and after the exercise in comparison with before the physical task $(p<0.05)$. The present result suggests that fitness level and physical exercise ameliorate the visual perceptual skil ls (PED) of the subjects.
\end{abstract}

\section{Introduction}

Performance, in most sports activities, depends not only on the efficiency of physiological and energetic processes but also on perceptive and informative skills. Despite the role of the visual perceptual skills (i.e., the perception of the distance) in the detection of relevant information to decide and to react in a dynamic environment [5], to date, little attention has been accorded to this skills. In this topic and to the best of our knowledge, several studies have examined the effect of the intensity and the effort duration on the cognitive performances [2, 3, 4]. However, very few studies have studied the effect of the sports practice on the perceptive mechanisms. In view of the above consideration, the present studies' results will provide relevant practical recommendations that could ameliorate the development of the visual perceptual skills.

Aim of the study: To examine the effect of moderate-intensity-exercise on the perception and the estimation of the egocentric distance (i.e., visual perceptual skills) on trained and untrained subjects. Hypothesis: (i) Visual perceptual skill represented by the estimation of the egocentric distance will be more accurate during and after than before the physical effort. (ii) Visual perceptual skill represented by the estimation of the egocentric distance will be accurate in trained than untrained subjects.

\section{Methods}

\section{Subjects}

Two groups of 18 male subjects volunteered to participate in the study: a group of soccer players $(23 \pm 0.5 \mathrm{yrs} ; 72 \pm 3.8 \mathrm{~kg} ; 1,73 \pm 2.5 \mathrm{~m})$ and a group of sedentary players $(23 \pm 0.2 \mathrm{yrs} ; 69 \pm 4.2 \mathrm{~kg}$; $1,71 \pm 2.8 \mathrm{~m}$ ) (do not take up a sports activity in teams).

\section{Procedure}

During the first part, maximal aerobic power of each subject was determined using an incremental test carried out on friction-loaded cycle-ergometer (Monark 894 ${ }^{\mathrm{E}}$, Stockholm, Sweden) performed until exhaustion: 5-min warm-up at $60 \mathrm{~W}$ followed by a continuous incremental test (i.e., intensity increment is $30 \mathrm{~W}$ ) with 2-min steps. During the second and third parts, each subject is called to estimate the distance (in meter) which separates him from a visual target put on gro und, before, during, and after an aerobic effort. During the exercise, estimations were performed at three time intervals (i.e., 1-2, 5-6 and 9-10 $\mathrm{min}$ ). These intervals were announced by the experimenter. Before starting the aerobic effort, subjects carries out $2 \mathrm{~min}$ of warm-up with resistance corresponding to $30 \%$ of his maximal aerobic power. Then, they pedal during $10 \mathrm{~min}$. The power corresponding to $50 \%$ of the power output in the last step of the

This is an Open Access article distributed under the terms of the Creative Commons Attribution-Noncommercial License 3.0, which permits unrestricted use, distribution, and reproduction in any noncommercial medium, provided the original work is properly cited. 
aerobic stress test was chosen as the effort intensity.

\section{Results}

There were significant main groups $\left(\mathrm{F}_{(1.34)}=9.4\right.$, $\mathrm{p}<0.01)$ and exercise $\left(\mathrm{F}_{(4.136)}=144.5, \mathrm{p}<0.001\right)$ effects. However, the distances effect was not significant $\left(\mathrm{F}_{(3.102)}=0.9, \mathrm{p}>0.05\right)$. The post-hoc test was significant indicating that the judgment of the distance was greater during and after the exercise with more precision in the trained than untrained subjects $(\mathrm{p}<0.05)$ (Figure 1). Moreover, the distances $\times$ exercise interaction $\left(\mathrm{F}_{(12.408)}=5.5\right.$, $\mathrm{p}<0.001)$ was significant demonstrating that the average estimations of the distances was affected by the duration of the physical effort (Figure 2). Generally, an underestimation of the real distances was especially observed at rest and in level 1 . However, the perceptive precisions (estimations of the distances) have improved from level 2. These performances get gradually closer, with the increase of the physical effort, the real distances, especially to level 3 and at the end of the effort. However, the ANOVA revealed that the groups $\times$ distances $\times$ exercise interaction $\left.\mathrm{F}_{(12.408)}=1.2, \mathrm{p}>0.05\right)$ was not significant.

\section{Discussion}

The results of the present study demonstrate that the perception of the egocentric distance depends on the physiological state of the body and on his awakening and activation level. Indeed, the estimation of the distance was more accurate during and after the exercise as observed by Proffitt et al.[1]. In addition, we have also shown that the perception of the egocentric distance depends on the fitness level of the subjects. In fact, the trained are better than the untrained on the estimation of the

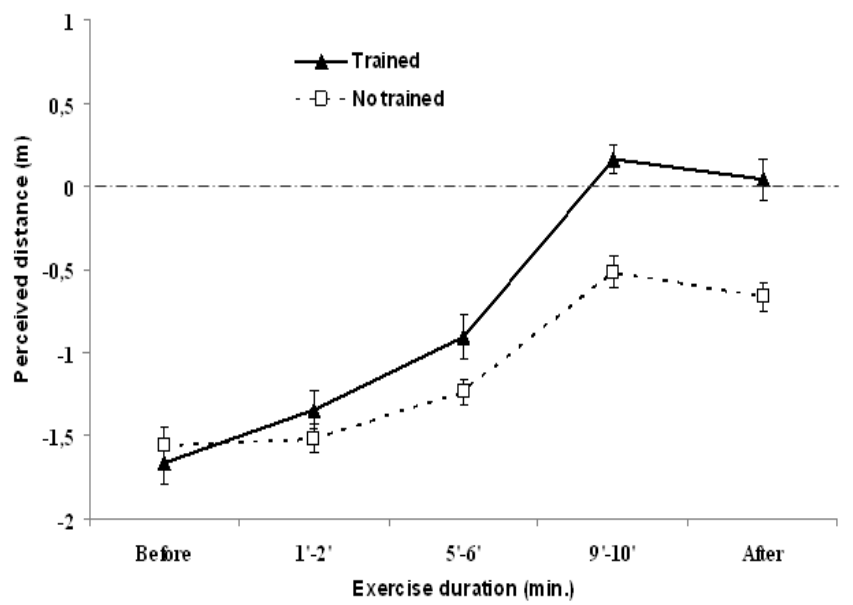

Figure 1. The estimation of the distances in the trained and the untrained subjects during the physical tasks. revealed that the estimation of the distances was significantly greater in trained than untrained subjects $(\mathrm{p}<0.01)$ and during and after the exercise in comparison with before the aerobic effort $(\mathrm{p}<0.05)$.

The interaction groups $\times$ exercise $\left(\mathrm{F}_{(4.136)}=9.7\right.$, $\mathrm{p}<0.001)$

distance during and at the end of the aerobic effort.

\section{Conclusion}

Visual perceptual skills should be developed for the trained subjects for better performances. Furthermore, this skill should be developed at rest and during physical exercise.

\section{References}

[1] D. Proffitt, J. Stefanucci, T. Banton, W. Epstein. The role of effort in distance perception. Psychological Science, 14, 106-113, 2003.

[2] J. Brisswalter, M. Durand, D. Delignières, P. Legros. Optimal and non-optimal demand in a dualtask of pedalling and simple reaction time: effects on energy expenditure and cognitive performance. Journal of Human Movement Studies, 29, 15-34, 1995.

[3] J. Brisswalter, R. Arcelin, M. Audiffren. Influence of physical exercise on simple reaction time performance: effect of physical fitness. Perceptual and Motor Skills, 85, 10-27, 1997.

[4] J.R. Thomas, D.M. Landers, W. Salazar, J. Etnier. Exercise and cognitive functioning. Physical activity fitness and health, 521-529. Champaign, IL: Human Kinetics.

[5] Z. Li, J. Phillips, F. Durgin. The underestimation of egocentric distance: evidence from frontal matching tasks. Atten Percept Psychophys, (In press), 2011.

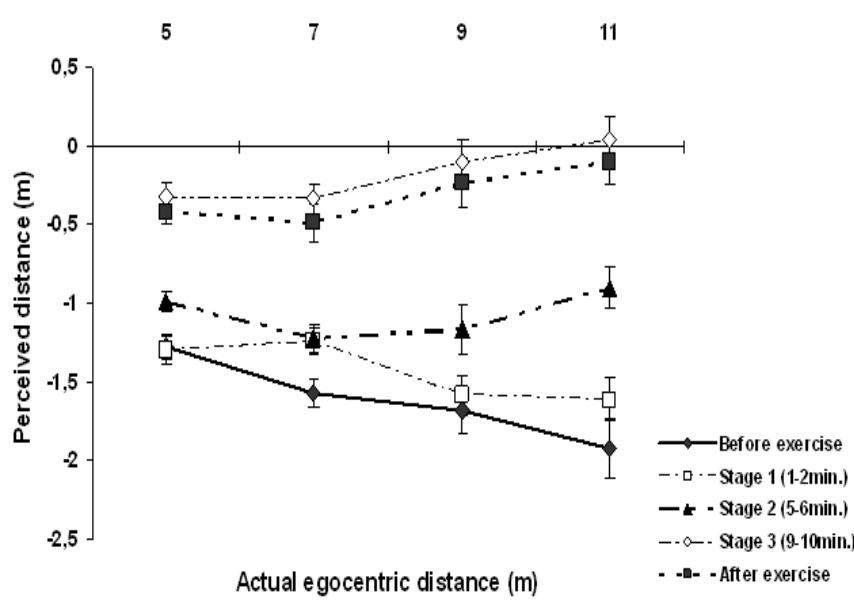

Figure 2. The estimations of distances according to the real distances before, during, and after the aerobic exercise. 